\title{
Effects of extended work shifts on employee fatigue, health, satisfaction, work/family balance, and patient safety
}

\author{
Madeleine Estryn-Béhara, Beatrice I.J.M. Van der Heijden ${ }^{\mathrm{b}}$, \& the NEXT Study Group \\ a Department of Occupational Health Hôtel Dieu, Assistance-Publique-Hôpitaux de Paris, , Place du Parvis \\ Notre Dame, 75004 Paris, France ; Tel : 33142348817 ; Fax 33142348520 , e-mail : madeleine.estryn- \\ behar@sap.aphp.fr

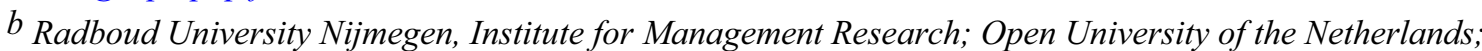 \\ University of Twente, the Netherlands.
}

\begin{abstract}
Europe. From our multivariate analysis concerning 25,924 European nurses, including twenty explanatory variables simultaneously, we found that work schedule itself is not a major determinant factor. Nurses aim to choose or accept night shifts or 12-hour shift in order to reduce their work/home conflicts, however, at the expense of the patient's safety, as well as their own health and safety. Therefore, it is important to develop measures, such as extended child care, association of nurses to the elaboration of their rota, 9- or 10-hour shifts in the afternoon, allowing naps during night shifts, and reduction of changing shifts with short notice. Work schedules must be organized in order to allow time for shift handover, social support and team building.
\end{abstract}

Keywords: Nurses, 12-hour shifts, Work/family balance, Burnout, Gender

\section{Introduction}

Work schedules should primarily be beneficial for employee fatigue, health, satisfaction, work/family balance, and patient safety. However, although previous research has found positive effects of 12hour shifts on employee satisfaction with working hours and free time, the impact of 12-hour shifts on employee fatigue, health and patient safety has mostly been non-significant or negative:

Recent evidence supports the negative impact of long work hours and its potential risks to nurses' health and to patient safety [3-4, 14-16]. However, 12-hour shifts are quickly spreading in Europe. In order to be able to formulate sound work schedule practices, a careful examination of shift work practices together with its internal system consequences, nurses' satisfaction, health and environmental factors is needed. The objective of our study was to perform a secondary analysis of a large
European data base, collected in 2003 [5-7], in order to determine the effect of work schedule among paramedical staff on these three parameters (work/family balance, health and safety), after adjustment for various risk factors.

\section{Methods}

\subsection{Procedure and Sample}

The NEXT study team has recruited, in ten European countries, health care institutions for participation in a survey aimed at a reflection of national distribution of nurses working in different types of institutions (hospitals, nursing homes, and home care), and across different geographical regions. Both the employer and the employee representative had to agree to participate. The completed questionnaires were returned to the 
national research institution by post, using a pre-paid envelope. In order to enhance ability to generalize, respondents were sampled across three different kinds of health care institutions, according to the specific repartition in each country: hospitals $(N=$ 147), nursing homes $(N=185)$, and home care institutions $(N=76)$. The survey was sent to 77,681 nurses, of whom 39,898 (51.7\%) responded. We obtained sufficient data about the work schedule of 25,924 nurses.

\subsection{Analyses}

Our primary interest was satisfaction with workschedules for well-being and for family life (single questions), and extent of Work-family conflict (W/FC) (five-item scale) [13]. As indicators for personal risk factors the following variables were included: Age; Seniority; Gender; Occupational level; Personal-family situation; Enough child care when at work; Satisfaction with pay (3-item scale).

As indicators for health problems we used several indicators, Burnout [10], Work-ability index [8], and single questions: How often do you feel tired? In periods when you are working, do you get sufficient sleep? Sleep quality on working days? Number of days of sick-leave declared.

As indicators for working conditions, the following variables were included: Being satisfied with staff handovers when shifts change; Being confronted with not knowing what a patient or a patient's family ought to be told about the patient's medical condition and its treatment; Have many interruptions and disturbances in the job; Being worried about making mistakes; Quantitative demand score indicating work intensity, measured using a 5-item scale based on the Copenhagen Psychosocial Questionnaire [11] and supplemented by a nursing specific item.; Physical load score, that we developed with 3 questions about 'lifting patients in bed without aid', 'maintaining an uncomfortable posture', and 'working in standing posture' [5], Quality of teamwork score that we developed [5-6]; Over-commitment score, measured with the short form of the Intrinsic Effort Scale [16] (5 items focus on the "inability to withdraw from work" and one item focuses on "disproportionate irritability."). More detailed information on the psychometric properties of the different variables used can be found in previous publications [5-7].

The Type of work schedule was calculated with the description of starting and ending time for each schedule. All types of part-time work were grouped in one category; Day length $7 \mathrm{~h}-8 \mathrm{~h} 30$ was grouped in the category ' 8 -hour shift'; Day 8h31-11h30 was grouped in the category ' 10 -hour shift'; Day $>11 \mathrm{~h} 31$ was grouped in the category '12-hour shift', similar categories were calculated for Night work; Alternating shifts were categorised in two groups, according to the number of nights worked per month $<6$ nights (mostly alternating morning and afternoon shift of 8 to 10 hours) or $\geq 6$ nights (mostly alternating 12-hour shift day and night). Also, several single questions were used: How many weekends (Saturday and/or Sunday) per month do you have to work? How often in one month do you have to get up before 5 o'clock in the morning to go to work? How often do you have to take over shifts with short notice? (1 to 3 days in advance)? How often in one month do you have a split shift?

For the major variables, bivariate tables were derived, using Pearson's Chi square test. Subsequently, for each dependent variable, a multivariate analysis using SPSS 12.0 software was performed (binary logistic regressions with 95\% confidence intervals). Overall, fewer than $10 \%$ of the different questions yielded missing data. For some variables that comprised several items, the missing data were the cause of the differences in sample sizes across the variables. We also reconducted the multivariate analysis stratified by gender [12] but no clear difference in results emerged. If not otherwise specified, all significant relationships reached a .001 significance level.

\section{Results}

\subsection{Work schedules and Work/family balance}

Nurses working part time but also nurses working 12-hour shift during the day and those working at night with 8 hour shift are a majority with low W/FC (Table1). The above groups, plus nurses working 12hour shift during the night report being satisfied with working time for their private life. On the other hand, nurses working alternating shifts and 10-hour shift at night report more difficulties with their private and family life. Extended work shifts are a solution for difficulties with child care, as they reduce the number of working days, which is efficient for nurses who have not enough childcare when at work. More female than male nurses work extended work shifts.

We must also note that $33.9 \%$ of male nurses work overtime, compare to $26.2 \%$ of the female nurses. 
Interestingly, only $23.3 \%$ of male nurses do the household chores themselves, compared to $72.7 \%$ of the female nurses. Furthermore, only $2.3 \%$ of male nurses live alone with one or more children, compared to $6.8 \%$ of the female nurses (and $9.9 \%$ of those working Night 12-h shift, 8.5\% Day 8- hour shift and 8.4\% Day10-hour shift). Male nurses with no children more frequently accept alternating shifts while female nurses with a child prefer 12-hour shifts during the day or night. Clearly nurses older than 45 try to leave alternating shifts. However, other aspects of work schedules influence family balance and they are reported less frequently by the nurses working extended work shifts. Indeed, nurses working 12hour shift during the day and the night are less frequently obliged to take over shifts at short notice, or to get up before 5 AM to go to work. Nurses working 12-hour night shifts report week-end work and split shifts less frequently.

Table 1

Work schedules of European nurses and work/family balance

\begin{tabular}{|c|c|c|c|c|c|c|c|c|c|c|c|}
\hline & & $\begin{array}{l}\text { Part } \\
\text { time }\end{array}$ & $\begin{array}{l}\text { Day } \\
8 \mathrm{~h}\end{array}$ & $\begin{array}{l}\text { Day } \\
10 \mathrm{~h}\end{array}$ & $\begin{array}{l}\text { Day } \\
12 \mathrm{~h}\end{array}$ & $\begin{array}{l}\text { Night } \\
8 \mathrm{~h}\end{array}$ & $\begin{array}{l}\text { Night } \\
10 \mathrm{~h}\end{array}$ & $\begin{array}{l}\text { Night } \\
12 \mathrm{~h}\end{array}$ & $\begin{array}{l}\text { Altern. } \\
<6 \mathrm{~N} / \mathrm{m}\end{array}$ & $\begin{array}{l}\text { Altern. } \\
\geq 6 \mathrm{~N} / \mathrm{m}\end{array}$ & Total \\
\hline \multirow{4}{*}{ Work/ family conflicts } & Low & $55.4 \%$ & $43.9 \%$ & $39.9 \%$ & $50.5 \%$ & $51.2 \%$ & $35.3 \%$ & $40.6 \%$ & $39.7 \%$ & $43.0 \%$ & $44.1 \%$ \\
\hline & Medium & $31.6 \%$ & $32.2 \%$ & $33.2 \%$ & $32.4 \%$ & $33.1 \%$ & $35.5 \%$ & $35.4 \%$ & $34.3 \%$ & $33.5 \%$ & $33.1 \%$ \\
\hline & High & $13.0 \%$ & $23.9 \%$ & $26.9 \%$ & $17.1 \%$ & $15.7 \%$ & $29.2 \%$ & $24.0 \%$ & $26.0 \%$ & $23.5 \%$ & $22.7 \%$ \\
\hline & $\mathrm{N}$ & 3847 & 7399 & 687 & 933 & 686 & 3478 & 483 & 3121 & 2686 & 23320 \\
\hline \multirow{3}{*}{$\begin{array}{l}\text { Satisfied w. working time } \\
\text { / private life }\end{array}$} & Yes & $74.6 \%$ & $64.1 \%$ & $63.5 \%$ & $66.4 \%$ & $64.4 \%$ & $59.6 \%$ & $69.2 \%$ & $50.3 \%$ & $56.6 \%$ & $62.6 \%$ \\
\hline & No & $25.4 \%$ & $35.9 \%$ & $36.5 \%$ & $33.6 \%$ & $35.6 \%$ & $40.4 \%$ & $30.8 \%$ & $49.7 \%$ & $43.4 \%$ & $37.4 \%$ \\
\hline & $\mathrm{N}$ & 3909 & 7489 & 1185 & 1182 & 679 & 3414 & 676 & 3522 & 2847 & 24903 \\
\hline \multirow{3}{*}{ Enough child care when at work } & Yes & $92.4 \%$ & $88.8 \%$ & $91.9 \%$ & $88.1 \%$ & $94.2 \%$ & $87.6 \%$ & $87.3 \%$ & $89.3 \%$ & $86.2 \%$ & $89.2 \%$ \\
\hline & No & $7.6 \%$ & $11.2 \%$ & $8.1 \%$ & $11.9 \%$ & $5.8 \%$ & $12.4 \%$ & $12.7 \%$ & $10.7 \%$ & $13.8 \%$ & $10.8 \%$ \\
\hline & $\mathrm{N}$ & 4040 & 7026 & 1145 & 1214 & 695 & 3528 & 675 & 3017 & 2857 & 24197 \\
\hline \multirow{4}{*}{ Age } & $<30$ years old & $21.3 \%$ & $16.7 \%$ & $17.1 \%$ & $20.1 \%$ & $28.9 \%$ & $17.5 \%$ & $15.9 \%$ & $25.8 \%$ & $23.8 \%$ & $20.1 \%$ \\
\hline & $30-45$ years old & $55.9 \%$ & $52.4 \%$ & $57.2 \%$ & $64.7 \%$ & $55.4 \%$ & $60.3 \%$ & $53.4 \%$ & $54.5 \%$ & $64.1 \%$ & $56.6 \%$ \\
\hline & $>45$ years old & $22.8 \%$ & $30.9 \%$ & $25.7 \%$ & $15.3 \%$ & $15.6 \%$ & $22.2 \%$ & $30.7 \%$ & $19.7 \%$ & $12.1 \%$ & $23.3 \%$ \\
\hline & $\mathrm{N}$ & 4035 & 7798 & 1213 & 1211 & 691 & 3574 & 698 & 3560 & 2902 & 25682 \\
\hline \multirow{3}{*}{ Gender } & female & $91.2 \%$ & $89.0 \%$ & $89.4 \%$ & $94.9 \%$ & $86.7 \%$ & $78.1 \%$ & $90.7 \%$ & $89.8 \%$ & $91.5 \%$ & $88.5 \%$ \\
\hline & male & $8.8 \%$ & $11.0 \%$ & $10.6 \%$ & $5.1 \%$ & $13.3 \%$ & $21.9 \%$ & $9.3 \%$ & $10.2 \%$ & $8.5 \%$ & $11.5 \%$ \\
\hline & $\mathrm{N}$ & 4044 & 7843 & 1222 & 1217 & 692 & 3599 & 702 & 3582 & 2917 & 25818 \\
\hline \multirow{4}{*}{$\begin{array}{l}\text { Family situation } \\
\text { Female nurses }\end{array}$} & With & $26.3 \%$ & $27.0 \%$ & $30.5 \%$ & $21.9 \%$ & $32.0 \%$ & $27.1 \%$ & $25.5 \%$ & $31.0 \%$ & $24.1 \%$ & $27.1 \%$ \\
\hline & Alone & $8.9 \%$ & $13.9 \%$ & $12.3 \%$ & $9.2 \%$ & $13.7 \%$ & $14.6 \%$ & $11.2 \%$ & $15.0 \%$ & $10.7 \%$ & $12.6 \%$ \\
\hline & e with child & $4.6 \%$ & $8.5 \%$ & $8.4 \%$ & $7.7 \%$ & $2.7 \%$ & $6.2 \%$ & $9.9 \%$ & $5.8 \%$ & $6.3 \%$ & $6.8 \%$ \\
\hline & With & $60.1 \%$ & $50.7 \%$ & $48.7 \%$ & $61.2 \%$ & $51.5 \%$ & $52.0 \%$ & $53.4 \%$ & $48.2 \%$ & $58.9 \%$ & $53.5 \%$ \\
\hline \multirow{4}{*}{$\begin{array}{l}\text { Family situation } \\
\text { Male nurses }\end{array}$} & With adult & $27.5 \%$ & $27.3 \%$ & $29.9 \%$ & $41.7 \%$ & $30.0 \%$ & $26.8 \%$ & $24.2 \%$ & $31.2 \%$ & $31.0 \%$ & $28.4 \%$ \\
\hline & Alon & $11.0 \%$ & $16.3 \%$ & $17.3 \%$ & $13.3 \%$ & $15.6 \%$ & $13.9 \%$ & $17.7 \%$ & $21.9 \%$ & $18.2 \%$ & $15.9 \%$ \\
\hline & Alone wi & $1.7 \%$ & $2.7 \%$ & $3.1 \%$ & $1.7 \%$ & $1.1 \%$ & $1.8 \%$ & $9.7 \%$ & $1.1 \%$ & $3.3 \%$ & $2.3 \%$ \\
\hline & With adult + child & $59.8 \%$ & $53.7 \%$ & $49.6 \%$ & $43.3 \%$ & $53.3 \%$ & $57.5 \%$ & $48.4 \%$ & $45.8 \%$ & $47.5 \%$ & $53.4 \%$ \\
\hline \multirow{3}{*}{\multicolumn{2}{|c|}{$\begin{array}{l}\text { How often do you have to take } 2 / / \text { month or less } \\
\text { over shifts at short notice? ( } 1 \text { to }>2 \text { / month } \\
3 \text { days in advance) }\end{array}$}} & $90.4 \%$ & $90.1 \%$ & $90.1 \%$ & $94.8 \%$ & $92.6 \%$ & $88.2 \%$ & $93.0 \%$ & $91.5 \%$ & $92.6 \%$ & $90.8 \%$ \\
\hline & & $9.6 \%$ & $9.9 \%$ & $9.9 \%$ & $5.2 \%$ & $7.4 \%$ & $11.8 \%$ & $7.0 \%$ & $8.5 \%$ & $7.4 \%$ & $9.2 \%$ \\
\hline & & 3503 & 6947 & 1135 & 1167 & 660 & 3281 & 645 & 3529 & 2852 & 23719 \\
\hline \multirow{4}{*}{$\begin{array}{l}\text { How often in one month do you } \\
\text { have to get up before } 5 \text { o'clock } \\
\text { in the morning to go to work? }\end{array}$} & No & $91.3 \%$ & $73.9 \%$ & $74.5 \%$ & $59.5 \%$ & $83.7 \%$ & $79.8 \%$ & $83.2 \%$ & $67.1 \%$ & $51.9 \%$ & $74.1 \%$ \\
\hline & $1-5 /$ month & $5.9 \%$ & $17.5 \%$ & $17.9 \%$ & $34.1 \%$ & $15.0 \%$ & $16.0 \%$ & $10.9 \%$ & $23.5 \%$ & $41.0 \%$ & $19.4 \%$ \\
\hline & $6+/$ month & $2.7 \%$ & $8.6 \%$ & $7.7 \%$ & $6.5 \%$ & $1.3 \%$ & $4.2 \%$ & $5.9 \%$ & $9.5 \%$ & $7.0 \%$ & $6.5 \%$ \\
\hline & $\mathrm{N}$ & 3877 & 7412 & 689 & 945 & 687 & 3505 & 488 & 3151 & 2728 & 23482 \\
\hline \multirow{3}{*}{$\begin{array}{l}\text { On how many weekends } \\
\text { (Saturday and/or Sunday) per } \\
\text { month do you have to work? }\end{array}$} & $2 / /$ month or less & $86.4 \%$ & $77.4 \%$ & $76.3 \%$ & $61.3 \%$ & $77.3 \%$ & $56.6 \%$ & $78.7 \%$ & $66.3 \%$ & $49.3 \%$ & $70.3 \%$ \\
\hline & $>2 /$ month & $13.6 \%$ & $22.6 \%$ & $23.7 \%$ & $38.7 \%$ & $22.7 \%$ & $43.4 \%$ & $21.3 \%$ & $33.7 \%$ & $50.7 \%$ & $29.7 \%$ \\
\hline & $\mathrm{N}$ & 3899 & 7475 & 1192 & 1206 & 683 & 3498 & 687 & 3568 & 2902 & 25110 \\
\hline \multirow{3}{*}{ Do you have split shift? } & No & $59.9 \%$ & $76.1 \%$ & $72.2 \%$ & $68.1 \%$ & $79.7 \%$ & $77.9 \%$ & $82.5 \%$ & $81.6 \%$ & $69.8 \%$ & $73.6 \%$ \\
\hline & Yes & $40.1 \%$ & $23.9 \%$ & $27.8 \%$ & $31.9 \%$ & $20.3 \%$ & $22.1 \%$ & $17.5 \%$ & $18.4 \%$ & $30.2 \%$ & $26.4 \%$ \\
\hline & $\mathrm{N}$ & 3334 & 6321 & 612 & 907 & 650 & 3078 & 429 & 3097 & 2654 & 21082 \\
\hline
\end{tabular}

\subsection{Work schedules, work content and Health}

Work organisation differs highly according to work schedules, and this influences W/FC (Table 2). Nurses working 10- or 12-hour shift at night are more often dissatisfied with staff handovers when shifts change. Nurses working 12-hour day and night shifts and those working alternating shifts including many nights often do not know what a patient or family ought to be told, they worry more about making mistakes and more report low quality of teamwork. More than $30 \%$ of day nurses show an high over- 
commitment score (at home, switch off not easy...). Nurses working 12-hour shifts during the day and those working alternating shifts report more interruptions and disturbances in the job, high quantitative demand and high physical load.
Even if nurses working 10- or 12-hour shifts state more often that they are satisfied with family life, their health is often worse, compared to nurses working 8-hour shift (Table 2 part 2).

Table 2

Work schedules of European nurses, work/content and health

\begin{tabular}{|c|c|c|c|c|c|c|c|c|c|c|c|}
\hline & & $\begin{array}{l}\text { Part } \\
\text { time }\end{array}$ & $\begin{array}{l}\text { Day } \\
8 \mathrm{~h} \\
\end{array}$ & $\begin{array}{l}\text { Day } \\
10 \mathrm{~h}\end{array}$ & $\begin{array}{l}\text { Day } \\
12 \mathrm{~h}\end{array}$ & $\begin{array}{l}\text { Night } \\
8 \mathrm{~h} \\
\end{array}$ & $\begin{array}{l}\text { Night } \\
10 \mathrm{~h}\end{array}$ & $\begin{array}{l}\text { Night } \\
12 \mathrm{~h}\end{array}$ & $\begin{array}{l}\text { Altern. } \\
<6 \mathrm{~N} / \mathrm{m}\end{array}$ & $\begin{array}{l}\text { Altern. } \\
\geq 6 \mathrm{~N} / \mathrm{m}\end{array}$ & Total \\
\hline \multirow{3}{*}{$\begin{array}{l}\text { Are you satisfied with } \\
\text { staff handovers when } \\
\text { shifts change? }\end{array}$} & Yes & $71.3 \%$ & $55.5 \%$ & $69.3 \%$ & $68.5 \%$ & $64.8 \%$ & $51.6 \%$ & $54.6 \%$ & $60.7 \%$ & $66.4 \%$ & $60.9 \%$ \\
\hline & No & $28.7 \%$ & $44.5 \%$ & $30.7 \%$ & $31.5 \%$ & $35.2 \%$ & $48.4 \%$ & $45.4 \%$ & $39.3 \%$ & $33.6 \%$ & $39.1 \%$ \\
\hline & $\mathrm{N}$ & 3548 & 6712 & 1125 & 1177 & 674 & 3346 & 643 & 3525 & 2878 & 23628 \\
\hline \multirow{3}{*}{$\begin{array}{l}\text { Not knowing what a } \\
\text { patient or family ought } \\
\text { to be told ab. patient's } \\
\text { condition and treatment }\end{array}$} & Never or sometimes & $77.7 \%$ & $69.3 \%$ & $73.0 \%$ & $69.2 \%$ & $78.7 \%$ & $71.9 \%$ & $66.2 \%$ & $70.2 \%$ & $62.2 \%$ & $70.6 \%$ \\
\hline & Often or always & $22.3 \%$ & $30.7 \%$ & $27.0 \%$ & $30.8 \%$ & $21.3 \%$ & $28.1 \%$ & $33.8 \%$ & $29.8 \%$ & $37.8 \%$ & $29.4 \%$ \\
\hline & $\mathrm{N}$ & 3880 & 7524 & 1180 & 1177 & 681 & 3489 & 668 & 3516 & 2873 & 24988 \\
\hline \multirow{3}{*}{$\begin{array}{l}\text { Worry about making } \\
\text { mistakes }\end{array}$} & seldom or sometimes & $84.6 \%$ & $73.7 \%$ & $73.4 \%$ & $59.3 \%$ & $81.7 \%$ & $71.2 \%$ & $66.0 \%$ & $75.6 \%$ & $64.9 \%$ & $73.7 \%$ \\
\hline & Often & $15.4 \%$ & $26.3 \%$ & $26.6 \%$ & $40.7 \%$ & $18.3 \%$ & $28.8 \%$ & $34.0 \%$ & $24.4 \%$ & $35.1 \%$ & $26.3 \%$ \\
\hline & $\mathrm{N}$ & 4018 & 7761 & 1212 & 1188 & 688 & 3563 & 694 & 3564 & 2848 & 25536 \\
\hline \multirow{3}{*}{$\begin{array}{l}\text { Overcommitment } \\
\text { score }\end{array}$} & Low & $76.6 \%$ & $68.6 \%$ & $66.6 \%$ & $68.9 \%$ & $84.1 \%$ & $71.7 \%$ & $70.0 \%$ & $73.1 \%$ & $70.0 \%$ & $71.6 \%$ \\
\hline & High & $23.4 \%$ & $31.4 \%$ & $33.4 \%$ & $31.1 \%$ & $15.9 \%$ & $28.3 \%$ & $30.0 \%$ & $26.9 \%$ & $30.0 \%$ & $28.4 \%$ \\
\hline & $\mathrm{N}$ & 3848 & 7388 & 685 & 939 & 685 & 3475 & 487 & 3115 & 2699 & 23321 \\
\hline \multirow{4}{*}{$\begin{array}{l}\text { Have many } \\
\text { interruptions and } \\
\text { disturbances in the job }\end{array}$} & No or not disturbing & $65.8 \%$ & $29.9 \%$ & $33.0 \%$ & $32.7 \%$ & $51.2 \%$ & $31.0 \%$ & $29.9 \%$ & $24.3 \%$ & $28.2 \%$ & $35.9 \%$ \\
\hline & derat. disturb. & $24.5 \%$ & $41.6 \%$ & $41.0 \%$ & $31.7 \%$ & $35.2 \%$ & $38.2 \%$ & $44.3 \%$ & $42.3 \%$ & $34.9 \%$ & $37.0 \%$ \\
\hline & yes considerab. dist. & $9.7 \%$ & $28.5 \%$ & $26.0 \%$ & $35.6 \%$ & $13.6 \%$ & $30.8 \%$ & $25.8 \%$ & $33.4 \%$ & $36.9 \%$ & $27.1 \%$ \\
\hline & $\mathrm{N}$ & 3869 & 7465 & 693 & 947 & 685 & 3522 & 488 & 3150 & 2713 & 23532 \\
\hline \multirow{4}{*}{ Quality of teamwork } & High & $61.4 \%$ & $37.4 \%$ & $41.1 \%$ & $30.7 \%$ & $47.4 \%$ & $31.7 \%$ & $35.2 \%$ & $36.1 \%$ & $27.8 \%$ & $39.4 \%$ \\
\hline & Medium & $32.2 \%$ & $48.4 \%$ & $44.8 \%$ & $49.9 \%$ & $43.1 \%$ & $47.5 \%$ & $49.5 \%$ & $50.0 \%$ & $50.7 \%$ & $45.9 \%$ \\
\hline & Low & $6.4 \%$ & $14.2 \%$ & $14.2 \%$ & $19.3 \%$ & $9.5 \%$ & $20.8 \%$ & $15.3 \%$ & $13.9 \%$ & $21.5 \%$ & $14.8 \%$ \\
\hline & $\mathrm{N}$ & 3670 & 6934 & 650 & 859 & 663 & 3275 & 463 & 2988 & 2525 & 22027 \\
\hline \multirow{4}{*}{ Quantitative demand } & Low & $14.4 \%$ & $12.1 \%$ & $10.4 \%$ & $6.8 \%$ & $13.6 \%$ & $11.7 \%$ & $11.6 \%$ & $7.3 \%$ & $6.2 \%$ & $10.8 \%$ \\
\hline & Medium & $56.1 \%$ & $50.0 \%$ & $48.6 \%$ & $48.9 \%$ & $58.0 \%$ & $51.6 \%$ & $50.6 \%$ & $47.6 \%$ & $42.9 \%$ & $50.2 \%$ \\
\hline & High & $29.5 \%$ & $37.9 \%$ & $41.0 \%$ & $44.3 \%$ & $28.4 \%$ & $36.8 \%$ & $37.7 \%$ & $45.0 \%$ & $50.9 \%$ & $39.1 \%$ \\
\hline & $\mathrm{N}$ & 4029 & 7793 & 1215 & 1203 & 691 & 3575 & 697 & 3573 & 2890 & 25666 \\
\hline \multirow{4}{*}{ Physical load score } & Low & $61.4 \%$ & $37.4 \%$ & $41.1 \%$ & $30.7 \%$ & $47.4 \%$ & $31.7 \%$ & $35.2 \%$ & $36.1 \%$ & $27.8 \%$ & $39.4 \%$ \\
\hline & Medium & $32.2 \%$ & $48.4 \%$ & $44.8 \%$ & $49.9 \%$ & $43.1 \%$ & $47.5 \%$ & $49.5 \%$ & $50.0 \%$ & $50.7 \%$ & $45.9 \%$ \\
\hline & High & $6.4 \%$ & $14.2 \%$ & $14.2 \%$ & $19.3 \%$ & $9.5 \%$ & $20.8 \%$ & $15.3 \%$ & $13.9 \%$ & $21.5 \%$ & $14.8 \%$ \\
\hline & $\mathrm{N}$ & 3686 & 6820 & 1130 & 1066 & 654 & 3287 & 616 & 3425 & 2654 & 23338 \\
\hline \multirow{3}{*}{$\begin{array}{l}\text { Satisfied w. working } \mathrm{T} \\
\text { / well being }\end{array}$} & Yes & $84.3 \%$ & $72.5 \%$ & $74.0 \%$ & $67.4 \%$ & $77.7 \%$ & $64.9 \%$ & $71.3 \%$ & $61.9 \%$ & $60.6 \%$ & $70.4 \%$ \\
\hline & No & $15.7 \%$ & $27.5 \%$ & $26.0 \%$ & $32.6 \%$ & $22.3 \%$ & $35.1 \%$ & $28.7 \%$ & $38.1 \%$ & $39.4 \%$ & $29.6 \%$ \\
\hline & $\mathrm{N}$ & 3960 & 7613 & 1196 & 1201 & 686 & 3483 & 680 & 3542 & 2879 & 25240 \\
\hline \multirow{3}{*}{$\begin{array}{l}\text { How often do you feel } \\
\text { tired? }\end{array}$} & seldom & $64.8 \%$ & $51.7 \%$ & $46.8 \%$ & $50.9 \%$ & $69.0 \%$ & $55.6 \%$ & $49.0 \%$ & $49.6 \%$ & $51.8 \%$ & $54.1 \%$ \\
\hline & 3 / week + & $35.2 \%$ & $48.3 \%$ & $53.2 \%$ & $49.1 \%$ & $31.0 \%$ & $44.4 \%$ & $51.0 \%$ & $50.4 \%$ & $48.2 \%$ & $45.9 \%$ \\
\hline & $\mathrm{N}$ & 4005 & 7790 & 1217 & 1204 & 687 & 3548 & 690 & 3564 & 2886 & 25591 \\
\hline \multirow{3}{*}{ Burnout } & Low & $83.6 \%$ & $67.7 \%$ & $67.6 \%$ & $67.4 \%$ & $90.0 \%$ & $73.4 \%$ & $66.3 \%$ & $71.7 \%$ & $67.6 \%$ & $72.1 \%$ \\
\hline & High & $16.4 \%$ & $32.3 \%$ & $32.4 \%$ & $32.6 \%$ & $10.0 \%$ & $26.6 \%$ & $33.7 \%$ & $28.3 \%$ & $32.4 \%$ & $27.9 \%$ \\
\hline & $\mathrm{N}$ & 3980 & 7738 & 1217 & 1185 & 688 & 3519 & 685 & 3545 & 2862 & 25419 \\
\hline \multirow{3}{*}{ Work ability index } & High & $81.6 \%$ & $70.3 \%$ & $72.9 \%$ & $64.7 \%$ & $86.0 \%$ & $74.9 \%$ & $70.1 \%$ & $74.0 \%$ & $65.2 \%$ & $72.9 \%$ \\
\hline & Low & $18.4 \%$ & $29.7 \%$ & $27.1 \%$ & $35.3 \%$ & $14.0 \%$ & $25.1 \%$ & $29.9 \%$ & $26.0 \%$ & $34.8 \%$ & $27.1 \%$ \\
\hline & $\mathrm{N}$ & 3664 & 6873 & 1109 & 1118 & 634 & 2930 & 613 & 3368 & 2714 & 23023 \\
\hline \multirow{4}{*}{$\begin{array}{l}\text { In periods of work, } \\
\text { quality of your sleep? }\end{array}$} & $\mathrm{Bad}$. & $10.9 \%$ & $18.4 \%$ & $19.3 \%$ & $17.6 \%$ & $13.0 \%$ & $19.5 \%$ & $21.6 \%$ & $19.4 \%$ & $21.6 \%$ & $17.7 \%$ \\
\hline & Moderate & $33.5 \%$ & $39.9 \%$ & $38.8 \%$ & $44.1 \%$ & $31.9 \%$ & $43.1 \%$ & $42.9 \%$ & $40.3 \%$ & $45.1 \%$ & $39.9 \%$ \\
\hline & Rather good & $55.6 \%$ & $41.7 \%$ & $41.9 \%$ & $38.2 \%$ & $55.2 \%$ & $37.4 \%$ & $35.5 \%$ & $40.3 \%$ & $33.3 \%$ & $42.3 \%$ \\
\hline & $\mathrm{N}$ & 3879 & 7456 & 688 & 947 & 687 & 3509 & 485 & 3129 & 2697 & 23477 \\
\hline \multirow{3}{*}{$\begin{array}{l}\text { In periods of work, do } \\
\text { you get } \\
\text { sleep? }\end{array}$} & Not .quite enough & $35.1 \%$ & $49.0 \%$ & $47.8 \%$ & $50.7 \%$ & $37.5 \%$ & $51.5 \%$ & $55.9 \%$ & $54.5 \%$ & $59.8 \%$ & $48.9 \%$ \\
\hline & Yes. almost yes & $64.9 \%$ & $51.0 \%$ & $52.2 \%$ & $49.3 \%$ & $62.5 \%$ & $48.5 \%$ & $44.1 \%$ & $45.5 \%$ & $40.2 \%$ & $51.1 \%$ \\
\hline & $\mathrm{N}$ & 3877 & 7449 & 688 & 940 & 685 & 3500 & 487 & 3124 & 2680 & 23430 \\
\hline \multirow{4}{*}{ Sick leave } & No sick-leave & $44.4 \%$ & $47.2 \%$ & $39.9 \%$ & $57.2 \%$ & $34.7 \%$ & $44.4 \%$ & $49.5 \%$ & $40.8 \%$ & $58.0 \%$ & $46.5 \%$ \\
\hline & $1 \_5$ & $28.0 \%$ & $23.6 \%$ & $32.7 \%$ & $17.9 \%$ & $39.5 \%$ & $26.2 \%$ & $25.2 \%$ & $31.1 \%$ & $14.5 \%$ & $25.4 \%$ \\
\hline & $>\overline{5}$ & $27.6 \%$ & $29.2 \%$ & $27.3 \%$ & $24.9 \%$ & $25.8 \%$ & $29.4 \%$ & $25.2 \%$ & $28.1 \%$ & $27.6 \%$ & $28.1 \%$ \\
\hline & $\mathrm{N}$ & 3854 & 7214 & 1179 & 1076 & 659 & 3342 & 646 & 3456 & 2678 & 24104 \\
\hline
\end{tabular}


One-third of Nurses working 12-hour shifts during the day, 10- hour shifts at night and alternating shifts are dissatisfied with their working time relative to their well being. Nurses working 10 - or 12-hour shifts during the day, 12-hour shifts at night and alternating shifts feel more often tired, and have more frequently a high burnout score. For nurses working 12-hour shifts during the day and alternating shifts $\geq 6$ nights, Work ability index is more frequently low. In periods of work, their quality and quantity of sleep is more frequently bad. Nurses working 12-hour shifts during the day and alternating shifts $\geq 6$ nights take short sick leaves less often, since they work less often, but require an equal number of long sick leaves.

\subsection{Multivariate analysis of determinants for family and personal satisfaction}

Among work schedules, it is 8- and 10-hour shifts at night and alternating morning and afternoon shifts $<6$ nights that are the greatest risk factors for dissatisfaction with working time, with respect to private life and well-being (Table 3). But, not having enough childcare when at work is more important than the work schedule and work content has the most importance.

Table 3

Multivariate analysis of Work schedules and other risk factors for work /Family conflicts and dissatisfaction with working time for private life and wellbeing, among European nurses

\begin{tabular}{|c|c|c|c|c|c|c|c|c|c|c|c|c|c|c|}
\hline & & \multicolumn{5}{|c|}{ Work / family conflicts } & \multicolumn{4}{|c|}{$\begin{array}{l}\text { Dissatisfied w. working time } \\
\text { / Private life } \\
\end{array}$} & \multicolumn{4}{|c|}{$\begin{array}{c}\text { Dissatisfied w. working time } \\
\text { / Wellbeing } \\
\end{array}$} \\
\hline & & $\mathrm{N}$ & adjOR* & $\mathrm{p}$ & $95 \%$ & $\mathrm{CI}$ & $\mathrm{N}$ & adjOR* & $\mathrm{p}$ & $95 \% \mathrm{CI}$ & $\mathrm{N}$ & adj OR* & $\mathrm{p}$ & $95 \% \mathrm{CI}$ \\
\hline \multirow{9}{*}{$\begin{array}{l}\text { Work } \\
\text { schedule }\end{array}$} & Part time & 2415 & 1.00 & & & & 2410 & 1.00 & & & 2650 & 1.00 & & \\
\hline & Day $8 \mathrm{~h}$ & 3705 & 1.07 & ns & 0.92 & 1.25 & 3671 & 1.28 & $* * *$ & 1.111 .49 & 4281 & 1.13 & & 0.971 .32 \\
\hline & Day $10 \mathrm{~h}$ & 353 & 1.10 & ns & 0.84 & 1.44 & 346 & 1.23 & $\mathrm{~ns}$ & 0.941 .60 & 429 & 1.09 & & 0.821 .44 \\
\hline & Day $12 \mathrm{~h}$ & 619 & 0.86 & ns & 0.67 & 1.09 & 613 & 0.87 & ns & 0.681 .11 & 677 & 1.17 & & 0.901 .51 \\
\hline & Night $8 \mathrm{~h}$ & 551 & 1.22 & ns & 0.98 & 1.52 & 548 & 1.36 & $* *$ & 1.091 .69 & 570 & 1.27 & & 1.011 .60 \\
\hline & Night $10 \mathrm{~h}$ & 2222 & 1.16 & ns & 0.99 & 1.35 & 2186 & 1.39 & $* * *$ & 1.191 .62 & 2447 & 1.19 & & 1.021 .39 \\
\hline & Night $12 \mathrm{~h}$ & 265 & 0.89 & ns & 0.66 & 1.21 & 264 & 0.97 & ns & 0.721 .30 & 317 & 1.24 & & 0.921 .67 \\
\hline & Alter $<6 \mathrm{~N} / \mathrm{m}$ & 1873 & 1.03 & ns & 0.88 & 1.22 & 1874 & 1.43 & $* * *$ & 1.221 .68 & 2125 & 1.22 & & 1.031 .45 \\
\hline & Alter $\geq 6 \mathrm{~N} / \mathrm{m}$ & 1947 & 0.97 & ns & 0.80 & 1.16 & 1927 & 1.16 & ns & 0.971 .38 & 2074 & 1.00 & & 0.831 .20 \\
\hline \multirow{2}{*}{$\begin{array}{l}\text { Enough child care } \\
\text { when at work }\end{array}$} & Yes or no answer & 12400 & 1.00 & & & & 12317 & 1.00 & & & 13859 & 1.00 & & \\
\hline & No & 1550 & 1.94 & $* * *$ & 1.69 & 2.24 & 1522 & 1.63 & $* * *$ & 1.451 .84 & 1711 & 1.36 & $* * *$ & 1.201 .54 \\
\hline \multirow{3}{*}{$\begin{array}{l}\text { Quality } \\
\text { of teamwork } \\
\text { score }\end{array}$} & High & 5186 & 1.00 & & & & 5145 & 1.00 & & & 5814 & 1.00 & & \\
\hline & Medium & 6531 & 1.61 & $* * *$ & 1.47 & 1.76 & 6480 & 1.54 & $* * *$ & 1.411 .69 & 7292 & 2.04 & $* * *$ & 1.842 .26 \\
\hline & Low & 2233 & 2.13 & $* * *$ & 1.84 & 2.47 & 2214 & 2.27 & $* * *$ & 1.992 .59 & 2464 & 4.18 & $* * *$ & 54.79 \\
\hline \multirow{2}{*}{$\begin{array}{l}\text { Overcommitment } \\
\text { score }\end{array}$} & Low & 10172 & 1.00 & & & & 10097 & 1.00 & & & 11309 & 1.00 & & \\
\hline & High & 3778 & 2.45 & $* * *$ & 2.21 & 2.71 & 3742 & 1.63 & $* * *$ & 1.491 .79 & 4261 & 1.30 & $* * *$ & 1.181 .42 \\
\hline \multirow{3}{*}{$\begin{array}{l}\text { Many interruptions } \\
\text { and disturbances } \\
\text { in the job }\end{array}$} & No or not disturb. & 4991 & 1.00 & & & & 4959 & 1.00 & & & 5529 & 1.00 & & \\
\hline & Moderately dist.. & 5115 & 1.66 & $* * *$ & 1.51 & 1.82 & 5065 & 1.41 & $* * *$ & 1.281 .55 & 5767 & 1.03 & & 0.931 .14 \\
\hline & $\begin{array}{l}\text { Considerably } \\
\text { disturbing }\end{array}$ & 3844 & 2.17 & $* * *$ & 1.92 & 2.45 & 3815 & 1.64 & $* * *$ & 1.461 .84 & 4274 & 1.34 & $* * *$ & 1.191 .50 \\
\hline \multirow{3}{*}{$\begin{array}{l}\text { Quantitative } \\
\text { demand score }\end{array}$} & Low & 1510 & 1.00 & & & & 1500 & 1.00 & & & & & & \\
\hline & Medium & 6928 & 1.30 & $* * *$ & 1.14 & 1.48 & 6882 & 1.28 & $* * *$ & 1.111 .48 & & & & \\
\hline & High & 5512 & 1.58 & $* * *$ & 1.36 & 1.83 & 5457 & 1.50 & $* * *$ & 1.281 .75 & & & & \\
\hline \multirow{3}{*}{$\begin{array}{l}\text { Get up before } \\
5 \text { o'clock }\end{array}$} & No & 9943 & 1.00 & & & & 9876 & 1.00 & & & 11201 & 1.00 & & \\
\hline & $1-5 /$ month & 3028 & 1.29 & $* * *$ & 1.16 & 1.43 & 2988 & 1.28 & $* * *$ & 1.161 .41 & 3270 & 1.15 & & 1.041 .28 \\
\hline & $6+/$ month & 979 & 1.48 & $* * *$ & 1.25 & 1.75 & 975 & 1.54 & $* * *$ & 1.331 .80 & 1099 & 1.12 & & 0.961 .31 \\
\hline \multirow{2}{*}{$\begin{array}{l}\text { Take over shifts } \\
\text { at short notice }\end{array}$} & $<+2 /$ month & 12525 & 1.00 & & & & 12435 & 1.00 & & & 14008 & 1.00 & & \\
\hline & $>2 /$ month & 1425 & 1.42 & $* * *$ & 1.23 & 1.64 & 1404 & 1.47 & $* * *$ & 1.291 .66 & 1562 & 1.15 & & 1.021 .31 \\
\hline \multirow{2}{*}{ Weekend work } & $<+2 /$ month & 9867 & 1.00 & & & & 9802 & 1.00 & & & & & & \\
\hline & $>2 /$ month & 4083 & 1.28 & $* * *$ & 1.15 & 1.43 & 4037 & 1.58 & $* * *$ & 1.421 .75 & & & & \\
\hline \multirow{2}{*}{ Split shifts } & No & 10208 & 1.00 & & & & 10146 & 1.00 & & & & & & \\
\hline & Yes & 3742 & 1.14 & $* *$ & 1.04 & 1.25 & 3693 & 1.27 & $* * *$ & 1.161 .39 & & & & \\
\hline \multirow{2}{*}{$\begin{array}{l}\text { Satisfied with staff } \\
\text { handovers }\end{array}$} & Yes & 8052 & 1.00 & & & & 7978 & 1.00 & & & 9011 & 1.00 & & \\
\hline & No & 5898 & 1.17 & $* * *$ & 1.07 & 1.27 & 5861 & 1.29 & $* * *$ & 1.191 .40 & 6559 & 1.10 & & 1.011 .20 \\
\hline \multirow{3}{*}{$\begin{array}{l}\text { Physical load } \\
\text { score }\end{array}$} & Low & 3492 & 1.00 & & & & 3466 & 1.00 & & & 4019 & 1.00 & & \\
\hline & Medium & 5180 & 1.05 & ns & 0.95 & 1.17 & 5134 & 1.15 & $* *$ & 1.031 .27 & 5697 & 1.09 & & 0.981 .22 \\
\hline & High & 5278 & 1.17 & $* *$ & 1.05 & 1.31 & 5239 & 1.14 & & 1.021 .27 & 5854 & 1.16 & & 1.041 .30 \\
\hline \multirow{2}{*}{ Gender } & Male & 1865 & 1.00 & & & & 1839 & 1.00 & & & 2040 & 1.00 & & \\
\hline & Female & 12085 & 1.31 & $* * *$ & 1.16 & 1.48 & 12000 & 1.12 & & 1.001 .25 & 13530 & 0.86 & $* *$ & 0.770 .96 \\
\hline
\end{tabular}

*Adjusted Odds ratios: adjusted also in the model on Country, Age, Seniority, Department of work, Occupational level, Family situation, Satisfaction with pay, $\mathrm{ns}=$ not significant; $*=\mathrm{p}<. .05 ; * *=\mathrm{p}<.01 ; * * *=\mathrm{p}<.001$ 
Nurses reporting a low quality of teamwork have twice as many conflicts and dissatisfaction. Nurses with a high score of over-commitment see their personal life deteriorate. Interruptions at work and quantitative job demands are also more important to explain W/FC and dissatisfaction with respect to private life and well-being. Other aspects of work schedule have to be taken into consideration: Get up before 5 o'clock Take over shifts at short notice; Weekend work; Split shifts; Satisfaction with staff handovers.

\subsection{Multivariate analysis of determinants for health and safety}

Among work schedules, extended work shifts is the greatest risk factor for burnout, low WAI and frequent worries about making mistakes (Table 4).

Work content is again of major importance. Nurses reporting a low quality of teamwork have three times as much burnout, low WAI and two thirds more worries about making mistakes. Nurses with a high score of over-commitment are nearly twice as exhausted and worry more about making mistakes. Interruptions at work and quantitative demands are also more important to explain burnout, low WAI and worries about making mistakes.

Table 4

Multivariate analysis of Work schedules and other risk factors for health and safety, among European nurses

\begin{tabular}{|c|c|c|c|c|c|c|c|c|c|c|c|c|c|}
\hline & & \multicolumn{4}{|c|}{ Burnout } & \multicolumn{4}{|c|}{ Work ability index } & \multicolumn{4}{|c|}{$\begin{array}{c}\text { Frequent Worries about } \\
\text { making Mistakes }\end{array}$} \\
\hline & & $\mathrm{N}$ & $\begin{array}{l}\text { adj } \\
\text { OR* }\end{array}$ & $\mathrm{p}$ & $95 \% \mathrm{CI}$ & $\mathrm{N}$ & $\begin{array}{l}\text { adj } \\
\text { OR* }\end{array}$ & $\mathrm{p}$ & $95 \% \mathrm{CI}$ & $\mathrm{N}$ & $\begin{array}{l}\text { adj } \\
\text { OR* }\end{array}$ & $\mathrm{p}$ & $95 \% \mathrm{CI}$ \\
\hline \multirow{9}{*}{$\begin{array}{l}\text { Work } \\
\text { schedule }\end{array}$} & Part time & 3028 & 1.00 & & & 2634 & 1.00 & & & 3483 & 1.00 & & \\
\hline & Day $8 \mathrm{~h}$ & 4581 & 1.14 & ns & $0.97 \quad 1.33$ & 3723 & 0.96 & $\mathrm{~ns}$ & $0.82 \quad 1.13$ & 5993 & 1.00 & ns & $0.87 \quad 1.15$ \\
\hline & Day $10 \mathrm{~h}$ & 446 & 1.21 & ns & $0.93 \quad 1.57$ & 372 & 0.91 & $\mathrm{~ns}$ & $0.69 \quad 1.21$ & 539 & 1.15 & ns & 0.901 .46 \\
\hline & Day $12 \mathrm{~h}$ & 669 & 1.06 & ns & $0.83 \quad 1.35$ & 624 & 0.89 & ns & $0.70 \quad 1.15$ & 771 & 1.15 & ns & 0.931 .42 \\
\hline & Night $8 \mathrm{~h}$ & 587 & 0.92 & ns & $0.65 \quad 1.31$ & 545 & 1.07 & ns & $0.80 \quad 1.43$ & 646 & 1.16 & ns & 0.901 .49 \\
\hline & Night $10 \mathrm{~h}$ & 2622 & 1.22 & * & 1.031 .46 & 2139 & 1.13 & ns & $0.94 \quad 1.34$ & 3038 & 1.00 & ns & 0.861 .16 \\
\hline & Night $12 \mathrm{~h}$ & 326 & 1.34 & * & $1.00 \quad 1.78$ & 273 & 1.11 & ns & $0.81 \quad 1.51$ & 412 & 1.45 & $* *$ & 1.131 .87 \\
\hline & Alte. $<6$ nights $/ \mathrm{m}$ & 1944 & 1.07 & ns & $0.90 \quad 1.29$ & 1849 & 0.94 & ns & $0.78 \quad 1.13$ & 2431 & 1.13 & ns & 0.961 .32 \\
\hline & Alter. $\geq 6 \mathrm{nN} / \mathrm{m}$ & 2061 & 1.10 & ns & $0.91 \quad 1.33$ & 1938 & 0.92 & ns & $0.76 \quad 1.12$ & 2304 & 1.05 & ns & $0.89 \quad 1.24$ \\
\hline \multirow{2}{*}{$\begin{array}{l}\text { Enough child care } \\
\text { when at work }\end{array}$} & Yes or no answer & 14467 & 1.00 & & & 12586 & 1.00 & & & & & & \\
\hline & No & 1797 & 1.37 & $* * *$ & $1.22 \quad 1.55$ & 1511 & 1.28 & $* * *$ & 1.131 .45 & & & & \\
\hline \multirow{3}{*}{$\begin{array}{l}\text { Quality } \\
\text { of teamwork } \\
\text { score }\end{array}$} & High & 6183 & 1.00 & & & 5397 & 1.00 & & & 7644 & 1.00 & & \\
\hline & Medium & 7539 & 1.93 & $* * *$ & 1.742 .14 & 6548 & 1.78 & $* * *$ & 1.601 .98 & 9019 & 1.25 & $* * *$ & 1.151 .37 \\
\hline & Low & 2542 & 3.27 & $* * *$ & $2.86 \quad 3.75$ & 2152 & 3.23 & $* * *$ & $2.80 \quad 3.74$ & 2954 & 1.67 & $* * *$ & 1.491 .87 \\
\hline \multirow{2}{*}{$\begin{array}{l}\text { Overcommitment } \\
\text { score }\end{array}$} & Low & 11739 & 1.00 & & & 10239 & 1.00 & & & 14103 & 1.00 & & \\
\hline & High & 4525 & 2.98 & 0.000 & $2.73 \quad 3.24$ & 3858 & 1.97 & $* * *$ & 1.792 .16 & 5514 & 1.88 & $* * *$ & 1.742 .03 \\
\hline \multirow{3}{*}{$\begin{array}{l}\text { Many interruptions } \\
\text { and disturbances } \\
\text { in the job }\end{array}$} & No or not disturb. & 5913 & 1.00 & $* * *$ & & 5169 & 1.00 & & & 7138 & 1.00 & & \\
\hline & Moderately dist.ur & 5948 & 1.27 & $* * *$ & $1.14 \quad 1.41$ & 5137 & 1.12 & $*$ & 1.001 .26 & 7189 & 1.19 & $* * *$ & 1.091 .31 \\
\hline & Considerably dist. & 4403 & 2.06 & $* * *$ & $1.83 \quad 2.32$ & 3791 & 1.49 & $* * *$ & $1.31 \quad 1.69$ & 5290 & 1.70 & $* * *$ & 1.541 .89 \\
\hline \multirow{3}{*}{$\begin{array}{l}\text { Quantitative } \\
\text { demand } \\
\text { score }\end{array}$} & Low & 1796 & 1.00 & & & 1530 & 1.00 & & & 2170 & 1.00 & & \\
\hline & Medium & 8150 & 1.10 & ns & $0.93 \quad 1.30$ & 7040 & 1.17 & ns & $0.99 \quad 1.39$ & 9889 & 1.31 & $* * *$ & $1.14 \quad 1.51$ \\
\hline & High & 6318 & 1.31 & $* *$ & 1.101 .56 & 5527 & 1.34 & $* * *$ & 1.121 .61 & 7558 & 1.68 & $* * *$ & 1.451 .94 \\
\hline \multirow{3}{*}{$\begin{array}{l}\text { get up before } \\
5 \text { o'clock }\end{array}$} & No & 11903 & 1.00 & & & & & & & & & & \\
\hline & $1-5 /$ month & 3276 & 1.03 & $\mathrm{~ns}$ & $0.93 \quad 1.14$ & & & & & & & & \\
\hline & $6+/$ month & 1085 & 1.39 & $* * *$ & 1.191 .61 & & & & & & & & \\
\hline \multirow{2}{*}{$\begin{array}{l}\text { Satisfied with staff } \\
\text { handovers }\end{array}$} & Yes & & & & & 8274 & 1.00 & & & & & & \\
\hline & No & & & & & 5823 & 1.18 & $* * *$ & $1.08 \quad 1.30$ & & & & \\
\hline \multirow{3}{*}{$\begin{array}{l}\text { Physical load } \\
\text { score }\end{array}$} & Low & 4663 & 1.00 & 0.000 & & 3805 & 1.00 & & & & & & \\
\hline & Medium & 5840 & 1.10 & ns & $0.98 \quad 1.23$ & 5148 & 1.11 & ns & $0.98 \quad 1.25$ & & & & \\
\hline & High & 5761 & 1.26 & $* * *$ & $1.12 \quad 1.42$ & 5144 & 1.39 & $* * *$ & $1.23 \quad 1.57$ & & & & \\
\hline \multirow{2}{*}{ Gender } & Male & 2131 & 1.00 & & & 1837 & 1.00 & & & & & & \\
\hline & Female & 14133 & 1.82 & $* * *$ & 1.592 .07 & 12260 & 1.36 & $* * *$ & 1.191 .57 & & & & \\
\hline
\end{tabular}

*Adjusted Odds ratios: adjusted also on Country, Age, Seniority, Department of work, Occupational level, Family situation, Satisfaction with pay, Weekend work; Split shifts. $\mathrm{ns}=$ not significant; $*=\mathrm{p}<. .05 ; * *=\mathrm{p}<.01 ; * * *=\mathrm{p}<.001$

\subsection{Multivariate analysis of determinants for stability in the job}

Among work schedules, 8- and 10-hour shifts at night and alternating shifts $<6$ nights are the greatest risk factor for Intent to change work setting (ITC); (Table 5). Work schedules have no significant influence for Sick leave $>5$ days and Intent to leave nursing (ITL). 
In this analysis also work content has the greatest influence. Nurses reporting a low quality of teamwork have four times as much ITC and seven times as much ITL. This is also the highest risk factor for sick leave. A high score of Over-commitment and frequent Interruptions at work are also more

Multivariate analysis of Work schedules and other risk factors for determinants for stability in the job, among European nurses

\begin{tabular}{|c|c|c|c|c|c|c|c|c|c|c|c|c|c|}
\hline & & \multicolumn{4}{|c|}{ Sick leave $>5$ days } & \multicolumn{4}{|c|}{$\begin{array}{c}\text { Intent to change } \\
\text { work setting (ITC) }\end{array}$} & \multicolumn{4}{|c|}{ Intent to leave nursing (ITL) } \\
\hline & & $\mathrm{N}$ & adj OR* & $\mathrm{p}$ & $95 \% \mathrm{CI}$ & $\mathrm{N}$ & adj OR* & $\mathrm{p}$ & $95 \% \mathrm{CI}$ & $\mathrm{N}$ & adj OR* & $\mathrm{p}$ & $95 \% \mathrm{CI}$ \\
\hline \multirow{9}{*}{$\begin{array}{l}\text { Work } \\
\text { schedule }\end{array}$} & Part time & 2373 & 1.38 & $* * *$ & 1.181 .61 & 2650 & 1.00 & & & 3041 & 1.00 & & \\
\hline & Day $8 \mathrm{~h}$ & 3717 & 1.12 & ns & 0.981 .29 & 4281 & 1.13 & ns & 0.971 .32 & 4942 & 0.97 & ns & 0.811 .16 \\
\hline & Day $10 \mathrm{~h}$ & 384 & 1.16 & ns & 0.891 .51 & 429 & 1.09 & ns & 0.821 .44 & 447 & 1.19 & ns & 0.871 .62 \\
\hline & Day $12 \mathrm{~h}$ & 545 & 1.12 & ns & $0.87 \quad 1.44$ & 677 & 1.17 & ns & 0.901 .51 & 710 & 0.92 & ns & 0.681 .25 \\
\hline & Night $8 \mathrm{~h}$ & 531 & 1.00 & ns & 0.801 .26 & 570 & 1.27 & $*$ & * 1.011 .60 & 582 & 1.23 & ns & 0.921 .65 \\
\hline & Night $10 \mathrm{~h}$ & 2170 & 1.00 & & & 2447 & 1.19 & $*$ & * 1.021 .39 & 2712 & 0.90 & ns & 0.751 .09 \\
\hline & Night $12 \mathrm{~h}$ & 257 & 1.05 & ns & 0.771 .44 & 317 & 1.24 & ns & 0.921 .67 & 356 & 1.31 & ns & 0.951 .82 \\
\hline & Alte. $<6$ nights $/$ m & 2136 & 0.99 & ns & $0.85 \quad 1.17$ & 2125 & 1.22 & $*$ & k 1.031 .45 & 1918 & 1.05 & ns & $0.85 \quad 1.29$ \\
\hline & Alter. $\geq 6 \mathrm{nN} / \mathrm{m}$ & 1880 & 1.19 & * & 1.001 .41 & 2074 & 1.00 & ns & 0.831 .20 & 2097 & 0.96 & ns & $0.77 \quad 1.20$ \\
\hline \multirow{2}{*}{$\begin{array}{l}\text { Enough child care } \\
\text { when at work }\end{array}$} & Yes or no answer & & & & & 13859 & 1.00 & & & 14934 & 1.00 & & \\
\hline & No & & & & & 1711 & 1.36 & $* * *$ & * 1.201 .54 & 1871 & 1.28 & $* * *$ & 1.111 .46 \\
\hline \multirow{3}{*}{$\begin{array}{l}\text { Quality } \\
\text { of teamwork } \\
\text { score }\end{array}$} & High & 5288 & 1.00 & & & 5814 & 1.00 & & & 6392 & 1.00 & & \\
\hline & Medium & 6561 & 1.11 & * & $1.01 \quad 1.22$ & 7292 & 2.04 & $* * *$ & k 1.842 .26 & 7758 & 2.81 & $* * *$ & 2.453 .23 \\
\hline & Low & 2144 & 1.38 & $* * *$ & 1.201 .58 & 2464 & 4.18 & $* * *$ & 3.654 .79 & 2655 & 7.23 & $* * *$ & 6.168 .48 \\
\hline \multirow{2}{*}{$\begin{array}{l}\text { Overcommitment } \\
\text { score }\end{array}$} & Low & & & & & 11309 & 1.00 & & & 12074 & 1.00 & & \\
\hline & High & & & & & 4261 & 1.30 & $* * *$ & * 1.181 .42 & 4731 & 1.46 & $* * *$ & 1.321 .62 \\
\hline \multirow{3}{*}{$\begin{array}{l}\text { Many interruptions } \\
\text { and disturbances } \\
\text { in the job }\end{array}$} & No or not disturb. & & & & & 5529 & 1.00 & & & 6121 & 1.00 & & \\
\hline & Moderately dist.ur & & & & & 5767 & 1.03 & ns & 0.931 .14 & 6143 & 0.99 & ns & 0.871 .13 \\
\hline & Considerably dist. & & & & & 4274 & 1.34 & $* * *$ & k 1.191 .50 & 4541 & 1.28 & $* * *$ & 1.111 .46 \\
\hline \multirow{3}{*}{$\begin{array}{l}\text { Quantitative } \\
\text { demand } \\
\text { score }\end{array}$} & Low & 1457 & 1.00 & & & & & & & & & & \\
\hline & Medium & 6986 & 1.17 & & 1.021 .35 & & & & & & & & \\
\hline & High & 5550 & 1.19 & & 1.021 .39 & & & & & & & & \\
\hline \multirow{3}{*}{$\begin{array}{l}\text { get up before } \\
5 \text { o'clock }\end{array}$} & No & & & & & 11201 & 1.00 & & & & & & \\
\hline & $1-5 /$ month & & & & & 3270 & 1.15 & $* *$ & * 1.041 .28 & & & & \\
\hline & $6+/$ month & & & & & 1099 & 1.12 & ns & 0.961 .31 & & & & \\
\hline \multirow{2}{*}{$\begin{array}{l}\text { Take over shifts } \\
\text { at short notice }\end{array}$} & $<+2 / \mathrm{m}$ & 12595 & 1.00 & & & 14008 & 1.00 & & & & & & \\
\hline & $>2 / \mathrm{m}$ & 1398 & 1.22 & $* * *$ & 1.081 .38 & 1562 & 1.15 & $*$ & * 1.021 .31 & & & & \\
\hline \multirow{2}{*}{ Weekend work } & $<+2 / \mathrm{m}$ & 9766 & 1.00 & & & & & & & & & & \\
\hline & $>2 / \mathrm{m}$ & 4227 & 1.13 & * & 1.021 .26 & & & & & & & & \\
\hline \multirow{2}{*}{ Split shifts } & No & 10370 & 1.00 & & & & & & & & & & \\
\hline & Yes & 3623 & 1.14 & $* *$ & 1.041 .24 & & & & & & & & \\
\hline \multirow{2}{*}{$\begin{array}{l}\text { Satisfied with staff } \\
\text { handovers }\end{array}$} & Yes & 8151 & 1.00 & & & 9011 & 1.00 & & & & & & \\
\hline & No & 5842 & 1.19 & $* * *$ & 1.091 .29 & 6559 & 1.10 & $*$ & * 1.011 .20 & & & & \\
\hline \multirow{3}{*}{$\begin{array}{l}\text { Physical load } \\
\text { score }\end{array}$} & Low & 3558 & 1.00 & & & 4019 & 1.00 & & & & & & \\
\hline & Medium & 5201 & 1.02 & ns & $0.92 \quad 1.14$ & 5697 & 1.09 & ns & 0.981 .22 & & & & \\
\hline & High & 5234 & 1.16 & $* *$ & 1.041 .30 & 5854 & 1.16 & $* *$ & * 1.041 .30 & & & & \\
\hline \multirow{2}{*}{ Gender } & Male & 1849 & 1.00 & & & 2040 & 1.00 & & & & & & \\
\hline & Female & 12144 & 1.13 & $*$ & $1.00 \quad 1.27$ & 13530 & 0.86 & $* *$ & $0.77 \quad 0.96$ & & & & \\
\hline
\end{tabular}

*Adjusted Odds ratios: adjusted also in the model on Country, Age, Seniority, Department of work, Occupational level, Family situation, Satisfaction with pay.

\section{Discussion}

From our multivariate analysis, which included twenty explanatory variables simultaneously, we found that work schedule itself is not a major determinant factor for the different aspects analysed. important to explain ITC and ITL. High quantitative demand, physical load, Take over shifts at short notice, Weekend work, Split shifts, and dissatisfaction with staff handovers contribute also significantly to Sick leave.

Table 5 
Nightly 8 - and 10-hour shifts and alternating shifts with $<6$ nights per month appeared to be detrimental to nurses' satisfaction with work duration and shift, for their private life, well-being and increase their intent to change work setting. Nightly 10 - and 12hour shifts appeared to be linked with more burnout and low Work ability index. Nightly 12-hour shifts increase by nearly $50 \%$ worries about making mistakes. Alternating shifts with $\geq 6$ nights per month appear to be linked with an increase in sick leave of more than 5 days.

The multivariate analysis demonstrated that inadequate work content and social support (low quality of teamwork, dissatisfaction with staff handovers, having many interruptions and disturbances in the job and over-commitment are the highest risk factors for health effects. They have much more influence in comparison to the impact of work schedules, for association with work/family conflicts as well as health problems.

We conclude that nurses tend to choose or accept these night shifts or 12-hour shifts in order to reduce their work/home conflicts (working only 3 shifts of 12 hours per week), however, at the expense of their health. These work schedules appear to be more often linked to burnout. Moreover, there are legitimate concerns about the safety of the employee and the patient in an extended work hour environment.

Indeed a recent very broad review by Wagstaff and Sigstad. (2011) concluded that work periods $>8$ hours carry an increased risk of accidents that cumulates, so that the increased risk of accidents after about 12 hours is twice the risk after 8 hours. Their analysis demonstrated also that shift work including nights carries a substantial risk of accidents. More specifically, Olds and Clarke (2010) demonstrated that, even in the case of voluntary overtime, each additional three hour period worked per week, past 40 hours, was associated with an average increase of $3 \%$ of reported wrong medication or dose administration errors and of $3 \%$ of needlestick injuries.

Although the implementation of 12-hour shifts seemed to be an answer to work/family conflicts for nurses, and, as such, responded to the recruitment problems for managers, the risk for health and quality of care has been highly underestimated. Therefore, it is important to develop measures, such as extended child care, association of nurses for the elaboration of their rota, 9- or 10-hour shifts in the afternoon, allowing naps during night shifts, and reduction of changing shifts at short notice and to study its effects.
Work schedules must be organized in order to allow time for shift handover, social support and team building.

\section{References}

[1] Baker GR, Norton PG, Flintoft V, et al, (2004), The Canadian Adverse Events Study: the incidence of adverse events among hospital patients in Canada. CMAJ, 170, 11, 1678-1686.

[2] Borritz, M., R. Rugulies, K. B. Christensen, E. Villadsen and T. S. Kristensen (2006). "Burnout as a predictor of self-reported sickness absence among human service workers: prospective findings from three year follow up of the PUMA study." Occup Environ Med 63(2): 98-106.

[3] Estryn-Béhar M. (1996), Ergonomie hospitalière, théorie et pratiques France, Éditions Estem, Paris, 568 pages

[4] Estryn-Behar M (1997) Encyclopedia of occupational health and safety fourth edition International labor office, Geneva, Work Schedules and Night Work in Health Care 97.22 - 97.26. ; Strain in health care work 97. 17-97.22.

[5] Estryn-Béhar M. (2008) Santé et satisfaction des soignants au travail en France et en Europe. Presses de 1'EHESP Rennes, 383 p.

[6] Estryn-Béhar M., Van der Heijden B., Oginska H., et al.. (2007) The Impact of Social Work Environment, Teamwork, Characteristics, Burnout, and Personal Factors Upon Intent to Leave Among European Nurses, Med Care, 45, 939-950.

[7] Hasselhorn H-M, Tackenberg P, Müller B-H, and the NEXTStudy group, (2003), Working conditions and intent to leave the profession, among nursing staff in Europe. Working Life Research Report 7: 2003, NIWL, Stockholm, 223-250.

[8] IIlmarinen J., Tuomi K., Klockars M. (1997) Changes in work ability of active employees over a 11-year period. Scand J Work Environ Health 23, 49-57.

[9] Jansen NWH, Kant IJ, van Amelsvoort LGPM, Kristensen TS, Swaen GMH, Nijhuis FJN, 2006, Work-family conflict as a risk factor for sickness absence. Occup Environ Med; 63, 488-494.

[10] Kristensen TS, Borritz M, (2001), Copenhagen Burnout Inventory: Normative data from a representative Danish population on Personal Burnout and results from the PUMA study on Personal Burnout, Work Burnout, and Client Burnout. National Institute of Occupational Health, Copenhagen, Denmark.

[11] Kristensen, T. S., H. Hannerz, A. Hogh and V. Borg (2005). "The Copenhagen Psychosocial Questionnaire: a tool for the assessment and improvement of the psychosocial work environment." Scand J Work Environ Health 31(6): 438-49.

[12] Messing K, Tissot F, Stock SR. (2009) Should studies of risk factors for musculoskeletal disorders be stratified by gender? Lesson from the 1998 Québec Health and Social Survey. Scand J Work Environ Health; 35(2):95-112.

[13] Netemeyer, R. G., Boles, J. S. McMurrian, R. (1996). Development and validation of work-family conflict and familywork conflict scales. Journal of Applied Psychology, 81, 400-410.

[14] Olds DM, Clarke SP (2010) The Effect of Work Hours on Adverse Events and Errors in Health Care. J Safety Res. 2010; 41(2):153-162)

[15] Phillips J, Beam S, Brinker A, et al. (2001) Retrospective analysis of mortalities associated with medication errors. Am J Health Syst Pharm. 1;58(19):1835-41.

[16] Siegrist J, Starke D, Chandola T, et al. The measurement of effort-reward imbalance at work: European comparisons. Soc Sci Med. 2004;58:1483-1499.

[17] Wagstaff AS, Sigstad Lie JA (2011). Shift and night work and long working hours - a systematic review of safety implications. Scand J Work Environ Health;37(3):173-185. 